\title{
Center Manifolds - Optimal Regularity for Nonuniformly Hyperbolic Dynamics ${ }^{1}$
}

\author{
Luis Barreira and Claudia Valls
}

\begin{abstract}
For sufficiently small perturbations with continuous derivative, we show how to establish the optimal regularity of invariant center manifolds when the linear equation admits a nonuniform exponential trichotomy. We also consider the general case of exponential growth rates given by an arbitrary function. This includes the usual exponential behavior as a very special case. Our proof uses the fiber contraction principle to establish the regularity property. We note that the argument also applies to sufficiently small linear perturbations, without further changes.
\end{abstract}

\section{Introduction}

It is widely recognized that center manifold theorems are powerful tools in the analysis of the behavior of nonhyperbolic dynamical systems. For example, let us consider the dynamics generated by the differential equation

$$
u^{\prime}=A(t) u+f(t, u)
$$

and let us assume that $f(t, 0)=0$ for every $t$. One can ask wether the behavior of the solutions of equation (1) in a neighborhood of zero somehow imitates the behavior of those of the linear equation

$$
u^{\prime}=A(t) u \text {. }
$$

This is certainly the case when equation (2) admits an exponential dichotomy. Indeed, by the Grobman-Hartman theorem, locally the two dynamics are topologically conjugate. On the other hand, when equation (2) has some elliptic directions one can still establish the existence of invariant center manifolds that are tangent to the subspace generated by these directions. However, the situation is not so simple anymore. Namely, the

\footnotetext{
Key words: nonuniform exponential trichotomies, center manifolds.
}

${ }^{1}$ Partially supported by FCT through CAMGSD, Lisbon. 
behavior on a center manifold substantially depends on the nonlinear perturbation, and in general the behavior of the dynamics on the manifold need not imitate the behavior on the subspace. On the other hand, this behavior essentially determines the stability of the system. We note that one is still able to show that along the hyperbolic directions locally the two dynamics are topologically conjugate, that is, one can partially linearize the nonlinear dynamics, namely along the hyperbolic directions.

Accordingly, the study of center manifolds plays a crucial role in determining the stability of solutions of a differential equation. Namely, when equation (2) has no unstable directions, all solutions converge exponentially to the center manifold. Therefore, the stability of the system is completely determined by the behavior on the center manifold. Thus, one often considers a reduction to the center manifold, and determines the quantitative behavior on it. This has also the advantage of reducing the dimension of the system. We refer the reader to the book [6] for details and references. In particular, using normal forms there is also the possibility of an appropriate description of the possible bifurcations. We note that since one needs to approximate the center manifolds to sufficiently high order, it is also important to discuss their smoothness, and to understand how to approximate them up to a given order.

We concentrate in this paper on the study of the smoothness of invariant center manifolds for nonautonomous differential equations. In its classical formulation, the center manifold theorem applies to flows for which the linear equation (2) admits a uniform exponential trichotomy. In particular, this means that the exponential estimates for the solutions are assumed to be independent of the initial time. The study of center manifolds can be traced back to the works of Pliss [19] and Kelley [15]. A very detailed exposition in the case of autonomous equations is given in [20], adapting results in [22]. See also $[16,21]$ for the case of equations in infinite-dimensional spaces. We refer the reader to $[8,9,10,20]$ for more details and further references. It is difficult to give an original reference for the first published version of a center manifold theorem in the nonautonomous case. Certainly, the modifications that are necessary to pass from autonomous to nonautonomous systems are not substantial. In contrast, our main goal is to weaken the condition concerning the existence of a uniform exponential trichotomy, to include a nonuniform exponential behavior. In this case the partial hyperbolicity can be spoiled exponentially along each solution as the initial time changes. We note that a principal motivation for weakening the assumption of uniform exponential behavior for equation (2) is that almost all trajectories of a smooth dynamical system preserving a finite invariant measure have a linear variational equation with a nonuniform exponential trichotomy (see [3] for more details and further references). 
As it is already mentioned above, we consider the general case of nonuniform exponential behavior. In this respect, our results are also a contribution to the theory of nonuniform hyperbolicity. We refer to [1] for a detailed exposition of the theory, which goes back to the landmark works of Oseledets [17] and particularly Pesin [18]. Since then it became an important part of the general theory of dynamical systems and a principal tool in the study of stochastic behavior. We note that the nonuniform hyperbolicity conditions can be expressed in terms of the Lyapunov exponents. Among the most important properties due to nonuniform hyperbolicity is the existence of stable and unstable invariant manifolds, and their absolute continuity property established by Pesin. The theory also describes the ergodic properties of dynamical systems with a finite invariant measure absolutely continuous with respect to the volume, and it expresses the Kolmogorov-Sinai entropy in terms of the Lyapunov exponents by Pesin's entropy formula. In another direction, combining the nonuniform hyperbolicity with the nontrivial recurrence guaranteed by the existence of a finite invariant measure, the fundamental work of Katok revealed a very rich and complicated orbit structure, including an exponential growth rate for the number of periodic points measured in terms of the topological entropy, and the approximation of the entropy of an invariant measure by uniformly hyperbolic horseshoes. We refer to the book [1] for details and references.

To the best of our knowledge, the first center manifold theorem on the existence of smooth center manifolds in the context of nonuniform exponential behavior was obtained in [2], where we established the $C^{k}$ regularity of the center manifolds. We note however that this is not an optimal result since we require the top $C^{k}$ derivative of the perturbation to be Lipschitz. This observation is the main motivation for the present work, and our main aim is precisely to obtain the optimal smoothness of the center manifolds. For simplicity of the exposition we consider only the $C^{1}$ case. The case of higher smoothness can also be considered although at the expense of rather involved computations, which essentially require a convenient framework in order to obtain the required estimates. Incidentally, in [2] our proof of the $C^{k}$ smoothness of the manifolds uses a single fixed point problem in an appropriate complete metric space. We use a result in [13], going back to a lemma of Henry in [14], which precisely allows us to establish the existence and simultaneously the regularity of the center manifolds using a single fixed point problem, instead of needing an additional fixed point problem for each higher-order derivative. Essentially, the result says that the closed unit ball in the space $C^{k, \delta}$ of functions of class $C^{k}$ with Hölder continuous $k$ th derivative with Hölder exponent $\delta$ is closed with respect to the $C^{0}$-topology. This allows us to consider contraction maps solely using the supremum norm instead of needing any norm involving also the derivatives. See [11] for a related approach in the particular case of uniform exponential 
behavior. In order to obtain the required estimates, we need sharp bounds for the derivatives of the central component of the solutions, and for the derivatives of the vector field along a given graph. For this we use a multivariate version of the Faà di Bruno formula in [12] for the derivatives of a composition.

Here we use an entirely different approach, which allows us to obtain the optimal smoothness of the center manifolds. Namely, we use the fiber contraction principle together with an elaboration of an argument sketched in [7] (although now in the nonuniform setting and for arbitrary growth rates) to establish the continuity of fiber contraction. This last step turns out to be the main technical difficulty of the proof. We briefly recall the fiber contraction principle. Given metric spaces $X$ and $Y=\left(Y, d_{Y}\right)$, we say that a transformation $S: X \times Y \rightarrow X \times Y$ of the form

$$
S(x, y)=(T(x), A(x, y))
$$

for some functions $T: X \rightarrow X$ and $A: X \times Y \rightarrow Y$ is a fiber contraction if there exists $\lambda \in(0,1)$ such that

$$
d_{Y}(A(x, y), A(x, \bar{y})) \leq \lambda d_{Y}(y, \bar{y})
$$

for every $x \in X$ and $y, \bar{y} \in Y$. We also say that a fixed point $x_{0} \in X$ of $T$ is attracting if for every $x \in X$ we have $T^{n}(x) \rightarrow x_{0}$ when $n \rightarrow \infty$.

Proposition 1 (Fiber contraction principle). If $S$ is a continuous fiber contraction, $x_{0} \in X$ is an attracting fixed point of $T$, and $y_{0} \in Y$ is a fixed point of $A\left(x_{0}, \cdot\right)$, then $\left(x_{0}, y_{0}\right)$ is an attracting fixed point of $S$.

We also follow partially arguments in [5] although now for arbitrary growth rates. Namely, we consider linear equations as in (1) that may exhibit central, stable and unstable behavior with respect to arbitrary growth rates $e^{c \rho(t)}$ determined by an arbitrary function $\rho$. The usual exponential behavior is included as a very special case when $\rho(t)=t$. These arbitrary growth rates include for example situations in which all Lyapunov exponents of equation (1) are infinite (either $+\infty$ or $-\infty$ ). Finally, we note that we have already established the existence of Lipschitz center manifolds for arbitrary growth rates in [4], and so we simply refer to that paper in the Lipschitz part of proof.

\section{Setup}

Let $A: \mathbb{R} \rightarrow M_{p}$ be a $C^{1}$ function, where $M_{p}$ is the set of $p \times p$ matrices. We consider the initial value problem

$$
u^{\prime}=A(t) u, \quad u(s)=u_{s}
$$

for each $s \in \mathbb{R}$ and $u_{s} \in X=\mathbb{R}^{p}$. Its unique solution is defined for every $t \in \mathbb{R}$, and we write it in the form $u(t)=T(t, s) u_{s}$, where $T(t, s)$ is 
the associated linear evolution operator. Now we introduce the notion of exponential trichotomy, considering both nonuniform exponential behavior, and growth rates given by an arbitrary function $\rho$. More precisely, given a strictly increasing differentiable function $\rho: \mathbb{R} \rightarrow \mathbb{R}$, we say that equation (3) admits a $\rho$-nonuniform exponential trichotomy if there exist continuous functions $P, Q_{1}, Q_{2}: \mathbb{R} \rightarrow M_{p}$, such that $P(t), Q_{1}(t)$ and $Q_{2}(t)$ are projections for each $t \in \mathbb{R}$ satisfying

$$
P(t)+Q_{1}(t)+Q_{2}(t)=\mathrm{Id},
$$

and

$$
P(t) T(t, s)=T(t, s) P(s), \quad Q_{i}(t) T(t, s)=T(t, s) Q_{i}(s), i=1,2
$$

for every $t, s \in \mathbb{R}$, and there exist constants

$$
\bar{a}<\underline{c} \leq \bar{c}<\underline{b} \text { and } \varepsilon, D>0,
$$

such that for every $t \geq s$ we have

$$
\begin{aligned}
\|T(t, s) P(s)\| & \leq D e^{\bar{c}(\rho(t)-\rho(s))+\varepsilon|\rho(s)|}, \\
\left\|T(t, s)^{-1} Q_{2}(t)\right\| & \leq D e^{-\underline{b}(\rho(t)-\rho(s))+\varepsilon|\rho(t)|},
\end{aligned}
$$

and for every $t \leq s$ we have

$$
\begin{aligned}
\|T(t, s) P(s)\| & \leq D e^{-\underline{c}(\rho(s)-\rho(t))+\varepsilon|\rho(s)|}, \\
\left\|T(t, s)^{-1} Q_{1}(t)\right\| & \leq D e^{\bar{a}(\rho(s)-\rho(t))+\varepsilon|\rho(t)|} .
\end{aligned}
$$

In this case we define center, stable and unstable subspaces for each $s \in$ $\mathbb{R}$ by

$$
E(s)=P(s) X \quad \text { and } \quad F_{i}(s)=Q_{i}(s) X, i=1,2 .
$$

Setting $\rho(t)=t$ we recover the notion of nonuniform exponential trichotomy (see [3]). We present an example taken from [4].

Example 1. Take $\beta>\delta>0$. For each $t \in \mathbb{R} \backslash\{0\}$ let

$$
A(t)=\left(\begin{array}{ccc}
\delta\left(\frac{\cos t-1}{2 \sqrt{|t|}}-\sqrt{|t|} \sin |t|\right) & 0 & 0 \\
0 & -a(t) & 0 \\
0 & 0 & a(t)
\end{array}\right)
$$

where

$$
a(t)=\frac{1}{2 \sqrt{|t|}} b(|t|)+\sqrt{|t|} b^{\prime}(|t|)-\frac{\delta}{2 \sqrt{|t|}} \sin |t|-\delta \sqrt{|t|} \cos t
$$

for some $C^{1}$ function $b: \mathbb{R}_{0}^{+} \rightarrow \mathbb{R}$ such that

$$
\lim _{t \rightarrow 0^{+}} \frac{b(t)}{\sqrt{t}}=0, \quad b \mid[1, \infty)=\beta,
$$


and $b^{\prime}(t)>0$ for every $t \in(0,1)$. Setting $A(0)=0$ we obtain a continuous function $\mathbb{R} \ni t \mapsto A(t)$. Then equation (3) admits a nonuniform exponential trichotomy for which the constant $\varepsilon$ in (4) cannot be taken equal to zero.

We also consider a $C^{1}$ function $f: \mathbb{R} \times X \rightarrow X$. We assume that $f(t, 0)=$ 0 for every $t \in \mathbb{R}$, and that there exists $\delta>0$ such that

$$
\left\|\frac{\partial f}{\partial u}(t, u)\right\| \leq \delta \rho^{\prime}(t) e^{-3 \varepsilon|\rho(t)|}
$$

for every $t \in \mathbb{R}$ and $u \in X$. In addition, we assume that $f(t, u)=0$ for every $t \in \mathbb{R}$ and $u \in X$ with $\|u\| \geq c$, for some constant $c>0$. Given $s \in \mathbb{R}$ and

we denote by

$$
u_{s}=\left(\xi, \eta_{1}, \eta_{2}\right) \in E(s) \times F_{1}(s) \times F_{2}(s),
$$

$$
\left(x(t), y_{1}(t), y_{2}(t)\right)=\left(x\left(t, s, u_{s}\right), y_{1}\left(t, s, u_{s}\right), y_{2}\left(t, s, u_{s}\right)\right),
$$

with values in $E(t) \times F_{1}(t) \times F_{2}(t)$, the unique solution of the initial value problem

$$
u^{\prime}=A(t) u+f(t, u), \quad u(s)=u_{s},
$$

which is equivalent to

$$
\begin{aligned}
x(t) & =T(t, s) \xi+\int_{s}^{t} P(t) T(t, s) f\left(\tau, x(\tau), y_{1}(\tau), y_{2}(\tau)\right) d \tau, \\
y_{i}(t) & =T(t, s) \eta_{i}+\int_{s}^{t} Q_{i}(t) T(t, s) f\left(\tau, x(\tau), y_{1}(\tau), y_{2}(\tau)\right) d \tau, \quad i=1,2 .
\end{aligned}
$$

One can show that each solution is defined for every $t \in \mathbb{R}$. Moreover, $u(t)=0$ is a solution of equation (8). For each $\tau \in \mathbb{R}$ we write

$$
\Psi_{\tau}^{\lambda}\left(s, u_{s}\right)=\left(s+\tau, x\left(s+\tau, s, u_{s}\right), y_{1}\left(s+\tau, s, u_{s}\right), y_{2}\left(s+\tau, s, u_{s}\right)\right) .
$$

This is the flow generated by the autonomous equation

$$
t^{\prime}=1, \quad u^{\prime}=A(t) u+f(t, u) .
$$

\section{Existence of center manifolds}

We formulate and prove our main result in this section. It establishes the existence of $C^{1}$ center manifolds for equation (8), or more precisely for the origin of equation (8). We emphasize that this is the optimal regularity.

The center manifolds are obtained as graphs over the center subspaces. We first describe the class of functions of which we consider the graphs. Let also $X$ be the space of continuous functions

$$
\phi=\left(\phi_{1}, \phi_{2}\right):\{(s, \xi) \in \mathbb{R} \times X: \xi \in E(s)\} \rightarrow X,
$$


such that $\phi(s, 0)=0$,

$$
\phi(s, E(s)) \subset F_{1}(s) \times F_{2}(s),
$$

and

$$
\|\phi(s, \xi)-\phi(s, \bar{\xi})\| \leq\|\xi-\bar{\xi}\|
$$

for every $s \in \mathbb{R}$ and $\xi, \bar{\xi} \in E(s)$. We can easily verify that $\mathcal{X}$ is a complete metric space with the distance

$$
d(\phi, \psi)=\sup \left\{\frac{\|\phi(s, \xi)-\psi(s, \xi)\|}{\|\xi\|}: s \in \mathbb{R} \text { and } \xi \in E(s) \backslash\{0\}\right\} .
$$

Given $\phi \in X$, we define

$$
V_{\phi}=\{(s, \xi, \phi(s, \xi)):(s, \xi) \in \mathbb{R} \times E(s)\} .
$$

The following is our main result.

Theorem 1. Let $A$ and $f$ be $C^{1}$ functions. If the equation $u^{\prime}=A(t) u$ admits a $\rho$-nonuniform exponential trichotomy with

$$
\bar{c}-\underline{b}+\varepsilon<0 \text { and } \bar{a}-\underline{c}+\varepsilon<0,
$$

$f(t, 0)=f(t, u)=0$ for every $t \in \mathbb{R}$ and $u \in X$ with $\|u\| \geq c$, for some constant $c>0$, and condition (7) holds, then provided that $\delta$ is sufficiently small there exists a unique $\phi \in \mathcal{X}$ such that

$$
\Psi_{\tau}\left(V_{\phi}\right)=V_{\phi} \quad \text { for every } \quad \tau \in \mathbb{R} .
$$

Moreover:

1. the function $\xi \mapsto \phi(s, \xi)$ is of class $C^{1}$ for each $s \in \mathbb{R}$, and $V_{\phi}$ is a $C^{1}$ manifold;

2. if $(\partial f / \partial u)(t, 0)=0$ for every $t \in \mathbb{R}$, then

$$
(\partial \phi / \partial \xi)(s, 0)=0 \quad \text { and } \quad T_{(s, 0)} V_{\phi}=\mathbb{R} \times E(s)
$$

for every $s \in \mathbb{R}$;

3. for each $s \in \mathbb{R}$ and $\xi, \bar{\xi} \in E(s)$, we have

$$
\begin{aligned}
& \left\|\Psi_{t-s}(s, \xi, \phi(s, \xi))-\Psi_{t-s}(s, \bar{\xi}, \phi(s, \bar{\xi}))\right\| \\
& \leq \begin{cases}2 D e^{(\bar{c}+2 \delta D)(\rho(t)-\rho(s))+\varepsilon|\rho(s)|}\|\xi-\bar{\xi}\|, & t \geq s, \\
2 D e^{(-\underline{c}+2 \delta D)(\rho(s)-\rho(t))+\varepsilon|\rho(s)|}\|\xi-\bar{\xi}\|, & t \leq s .\end{cases}
\end{aligned}
$$

Proof. We note that property (12) is equivalent to the identities

$$
\begin{gathered}
x(t)=T(t, s) \xi+\int_{s}^{t} P(t) T(t, \tau) f(\tau, x(\tau), \phi(\tau, x(\tau))) d \tau, \\
\phi_{i}(t, x(t))=T(t, s) \phi_{i, \lambda}(s, \xi)+\int_{s}^{t} Q_{i}(t) T(t, \tau) f(\tau, x(\tau), \phi(\tau, x(\tau))) d \tau
\end{gathered}
$$


for every $i=1,2$, and $t \in \mathbb{R}$. The following statement establishes the existence of a solution of the first equation in (15) for each given $\phi$.

Lemma 1. For each $\phi \in X$ and $(s, \xi) \in \mathbb{R} \times E(s)$ there exists a unique continuous function

$$
x_{\phi}:\{(s, \xi) \in \mathbb{R} \times X: \xi \in E(s)\} \rightarrow X
$$

with $x_{\phi}(s, \xi)=\xi$ and $x_{\phi}(t, \xi) \in E(t)$ for each $t \in \mathbb{R}$, satisfying the first identity in (15) for every $t \in \mathbb{R}$. Moreover,

$$
\left\|x_{\phi}(t, \xi)\right\| \leq \begin{cases}2 D e^{(\bar{c}+2 \delta D)(\rho(t)-\rho(s))+\varepsilon|\rho(s)|}\|\xi\|, & t \geq s \\ 2 D e^{(-\underline{c}+2 \delta D)(\rho(s)-\rho(t))+\varepsilon|\rho(s)|}\|\xi\|, & t \leq s\end{cases}
$$

Lemma 1 follows from [4, Lemmas 1 and 2] (setting $\phi=\psi$ and $\bar{\xi}=0$ in the second lemma). Since

$$
\Psi_{t-s}(s, \xi, \phi(s, \xi))=\left(t, x_{\phi}(t, \xi), \phi\left(t, x_{\phi}(t, \xi)\right)\right),
$$

the inequalities in (14) follow readily from Lemma 1 and (9).

We have also the following estimates.

Lemma 2 ([4, Lemma 2$])$. For every $\phi, \psi \in \mathcal{X}, s \in \mathbb{R}$ and $\xi, \bar{\xi} \in E(s)$, we have

$$
\begin{aligned}
& \left\|x_{\phi}(t, \xi)-x_{\phi}(t, \bar{\xi})\right\|+\left\|x_{\phi}(t, \xi)-x_{\psi}(t, \xi)\right\| \\
& \quad \leq\left\{\begin{array}{l}
D e^{(\bar{c}+2 \delta D)(\rho(t)-\rho(s))+\varepsilon|\rho(s)|} \\
\times\left(\|\xi-\bar{\xi}\|+\frac{1}{2} e^{4 \delta D(\rho(t)-\rho(s))}\|\xi\| d(\phi, \psi)\right), \quad t \geq s, \\
D e^{(-\underline{c}+2 \delta D)(\rho(s)-\rho(t))+\varepsilon|\rho(s)|} \\
\times\left(\|\xi-\bar{\xi}\|+\frac{1}{2} e^{4 \delta D(\rho(t)-\rho(s))}\|\xi\| d(\phi, \psi)\right), \quad t \leq s .
\end{array}\right.
\end{aligned}
$$

We notice that $x_{\phi}$ is the unique solution of the equation

$$
x^{\prime}=A(t) x+P(t) f(t, x, \phi(t, x))
$$

with initial condition $x(s)=\xi$. By Lemma 2 and the continuous dependence of the solutions of a differential equation on the initial conditions, the function $(t, \phi, s, \xi) \mapsto x_{\phi}(t, \xi)$ is continuous.

Now we consider the two remaining equations in (15), and we rewrite them in a more convenient form.

Lemma 3. For every sufficiently small $\delta>0$, given $\phi \in X$ the following properties are equivalent: 
1. for every $s, t \in \mathbb{R}, \xi \in E(s)$, and $i=1,2$ we have

$$
\begin{aligned}
\phi_{i}\left(t, x_{\phi}(t, \xi)\right)= & T(t, s) \phi_{i}(s, \xi) \\
& +\int_{s}^{t} Q_{i}(t) T(t, \tau) f\left(\tau, x_{\phi}(\tau, \xi), \phi\left(\tau, x_{\phi}(\tau, \xi)\right)\right) d \tau ;
\end{aligned}
$$

2. for every $s \in \mathbb{R}$ and $\xi \in E(s)$ we have

$$
\begin{aligned}
& \phi_{1}(s, \xi)=\int_{-\infty}^{s} Q_{1}(s) T(\tau, s)^{-1} f\left(\tau, x_{\phi}(\tau, \xi), \phi\left(\tau, x_{\phi}(\tau, \xi)\right)\right) d \tau, \\
& \phi_{2}(s, \xi)=-\int_{s}^{\infty} Q_{2}(s) T(\tau, s)^{-1} f\left(\tau, x_{\phi}(\tau, \xi), \phi\left(\tau, x_{\phi}(\tau, \xi)\right)\right) d \tau .
\end{aligned}
$$

We can then show that there is a unique function $\phi$ satisfying the two identities in (17).

Lemma 4 ([4, Lemma 4]). Provided that $\delta$ is sufficiently small, there exists a unique $\phi \in \mathcal{X}$ such that (17) holds for every $s \in \mathbb{R}$ and $\xi \in E(s)$.

The function $\phi$ in Lemma 4 is the unique fixed point of a contraction operator $T$ in the space $X$. Namely, the operator is given by

$$
\begin{aligned}
(T \phi)(s, \xi)= & \left(\int_{-\infty}^{s} Q_{1}(s) T(\tau, s)^{-1} f\left(\tau, x_{\phi}(\tau, \xi), \phi\left(\tau, x_{\phi}(\tau, \xi)\right)\right) d \tau\right. \\
& \left.-\int_{s}^{\infty} Q_{2}(s) T(\tau, s)^{-1} f\left(\tau, x_{\phi}(\tau, \xi), \phi\left(\tau, x_{\phi}(\tau, \xi)\right)\right) d \tau\right)
\end{aligned}
$$

for each $\phi \in X$.

Now we establish the $C^{1}$ regularity of the map $\xi \mapsto \phi(s, \xi)$ for each $s \in \mathbb{R}$. This is the most delicate part of the proof (and this would still be the case without a nonuniform exponential behavior or an arbitrary function $\rho$ ). The strategy is to consider operators obtained from taking formally the derivative with respect to $\xi$ in the two equations in (17), and then show that these have fixed points in an appropriate space of continuous functions. The final step is to show that these fixed points are indeed obtained from derivating $\phi$, and thus $\phi$ must be of class $C^{1}$. The main difficulty that prevents one to show that the new operators considered together with $T$ in (18) are contractions is that we would need further regularity assumptions on $f$, and thus we would loose the optimal regularity of the center manifold. Instead we use the fiber contraction principle, which requires weaker assumptions. Nevertheless, one still needs to establish the continuity of the fiber contraction. This turns out to be the main technical difficulty of the proof. 
We consider the space $\mathcal{F}$ of continuous functions

$$
\Phi:\{(s, \xi) \in \mathbb{R} \times X: \xi \in E(s)\} \rightarrow \coprod_{s \in \mathbb{R}} L(s),
$$

where $L(s)$ is the family of linear transformations from $E(s)$ to $F_{1}(s) \times$ $F_{2}(s)$, such that $\Phi(s, \xi) \in L(s)$ for every $(s, \xi) \in \mathbb{R} \times E(s)$, with

$$
\|\Phi\|:=\sup \{\|\Phi(s, \xi)\|:(s, \xi) \in \mathbb{R} \times E(s)\} \leq 1 .
$$

We also consider the subset $\mathcal{F}_{0} \subset \mathcal{F}$ composed of the functions $\Phi \in \mathcal{F}$ such that $\Phi(s, 0)=0$ for every $s \in \mathbb{R}$. Clearly, $\mathcal{F}$ and $\mathcal{F}_{0}$ are complete metric spaces with the distance induced by the norm in (19).

We define a linear operator

$$
A(\phi, \Phi)=\left(A_{1}(\phi, \Phi), A_{2}(\phi, \Phi)\right)
$$

for each $(\phi, \Phi) \in \mathcal{X} \times \mathcal{F}$ by

$$
\begin{aligned}
& A_{1}(\phi, \Phi)(s, \xi) \\
& =\int_{-\infty}^{s} Q_{1}(s) T(\tau, s)^{-1}\left(\frac{\partial f}{\partial x}\left(y_{\phi}(\tau)\right) W(\tau)+\frac{\partial f}{\partial y}\left(y_{\phi}(\tau)\right) \Phi\left(z_{\phi}(\tau)\right) W(\tau)\right) d \tau
\end{aligned}
$$

and

$$
\begin{aligned}
& A_{2}(\phi, \Phi)(s, \xi) \\
& =-\int_{s}^{\infty} Q_{2}(s) T(\tau, s)^{-1}\left(\frac{\partial f}{\partial x}\left(y_{\phi}(\tau)\right) W(\tau)+\frac{\partial f}{\partial y}\left(y_{\phi}(\tau)\right) \Phi\left(z_{\phi}(\tau)\right) W(\tau)\right) d \tau,
\end{aligned}
$$

where $(x, y) \in E(\tau) \times\left(F_{1}(\tau) \times F_{2}(\tau)\right)$, with the notation

$$
y_{\phi}(t)=\left(t, x_{\phi}(t, \xi), \phi\left(t, x_{\phi}(t, \xi)\right)\right) \quad \text { and } \quad z_{\phi}(t)=\left(t, x_{\phi}(t, \xi)\right) \text {, }
$$

and where the linear operators

$$
W(t)=W_{\phi, \Phi, \xi}(t): E(s) \rightarrow E(t)
$$

are determined by the identities

$$
\begin{aligned}
W(t)= & P(t) T(t, s) \\
& +\int_{s}^{t} P(t) T(t, \tau)\left(\frac{\partial f}{\partial x}\left(y_{\phi}(\tau)\right) W(\tau)+\frac{\partial f}{\partial y}\left(y_{\phi}(\tau)\right) \Phi\left(z_{\phi}(\tau)\right) W(\tau)\right) d \tau
\end{aligned}
$$

for $t \in \mathbb{R}$. It follows from the continuity of the solutions of a differential equation with respect to parameters, and the continuity of the functions $(t, \phi, s, \xi) \mapsto x_{\phi}(t, \xi), \phi$, and $\Phi$, that the function $(t, \phi, s, \xi) \mapsto W_{\phi, \Phi, \xi}(t)$ is also continuous. 
Lemma 5. The operator $A$ is well-defined, and $A(X \times \mathcal{F}) \subset \mathcal{F}$.

Proof of the lemma. Set

$B_{1}=\int_{-\infty}^{s}\left\|Q_{1}(s) T(\tau, s)^{-1}\left(\frac{\partial f}{\partial x}\left(y_{\phi}(\tau)\right) W(\tau)+\frac{\partial f}{\partial y}\left(y_{\phi}(\tau)\right) \Phi\left(z_{\phi}(\tau)\right) W(\tau)\right)\right\| d \tau$,

and

$B_{2}=\int_{s}^{\infty}\left\|Q_{2}(s) T(\tau, s)^{-1}\left(\frac{\partial f}{\partial x}\left(y_{\phi}(\tau)\right) W(\tau)+\frac{\partial f}{\partial y}\left(y_{\phi}(\tau)\right) \Phi\left(z_{\phi}(\tau)\right) W(\tau)\right)\right\| d \tau$.

It follows from (6) and (7) that

$$
\begin{aligned}
B_{1} & \leq 2 \delta D \int_{-\infty}^{s} \rho^{\prime}(\tau) e^{\bar{a}(\rho(s)-\rho(\tau))+\varepsilon|\rho(\tau)|-3 \varepsilon|\rho(\tau)|}\|W(\tau)\| d \tau \\
& =2 \delta D \int_{-\infty}^{s} \rho^{\prime}(\tau) e^{\bar{a}(\rho(s)-\rho(\tau))-2 \varepsilon|\rho(\tau)|}\|W(\tau)\| d \tau
\end{aligned}
$$

Furthermore, it follows from (5) and (7) that

$$
\begin{aligned}
B_{2} & \leq 2 \delta D \int_{s}^{\infty} \rho^{\prime}(\tau) e^{-\underline{b}(\rho(\tau)-\rho(s))+\varepsilon|\rho(\tau)|-3 \varepsilon|\rho(\tau)|}\|W(\tau)\| d \tau \\
& =2 \delta D \int_{s}^{\infty} \rho^{\prime}(\tau) e^{-\underline{b}(\rho(\tau)-\rho(s))-2 \varepsilon|\rho(\tau)|}\|W(\tau)\| d \tau .
\end{aligned}
$$

On the other hand, by (22) and again (7), for $t \geq s$ we have

$$
\begin{aligned}
\|W(t)\| \leq & D e^{\bar{c}(\rho(t)-\rho(s))+\varepsilon|\rho(s)|} \\
& +2 \delta D \int_{s}^{t} \rho^{\prime}(\tau) e^{\bar{c}(\rho(t)-\rho(\tau))+\varepsilon|\rho(\tau)|-3 \varepsilon|\rho(\tau)|}\|W(\tau)\| d \tau
\end{aligned}
$$

Setting $\Gamma(t)=e^{-\bar{c}(\rho(t)-\rho(s))}\|W(t)\|$ we obtain

$$
\begin{aligned}
\Gamma(t) & \leq D e^{\varepsilon|\rho(s)|}+2 \delta D \int_{s}^{t} \rho^{\prime}(\tau) e^{-2 \varepsilon|\rho(\tau)|} \Gamma(\tau) d \tau \\
& \leq D e^{\varepsilon|\rho(s)|}+2 \delta D \int_{s}^{t} \rho^{\prime}(\tau) \Gamma(\tau) d \tau .
\end{aligned}
$$

It follows from Gronwall's lemma that

$$
\Gamma(t) \leq D e^{\varepsilon|\rho(s)|} e^{2 \delta D(\rho(t)-\rho(s))},
$$

and thus,

$$
\|W(t)\| \leq D e^{\varepsilon|\rho(s)|} e^{(\bar{c}+2 \delta D)(\rho(t)-\rho(s))}, \quad t \geq s .
$$

Proceeding in a similar manner, we find that

$$
\|W(t)\| \leq D e^{\varepsilon|\rho(s)|} e^{(-\underline{c}+2 \delta D)(\rho(s)-\rho(t))}, \quad t \leq s .
$$


Indeed, by (22) and again (7), for $t \leq s$ we have

$$
\begin{aligned}
\|W(t)\| \leq & D e^{-\underline{c}(\rho(s)-\rho(t))+\varepsilon|\rho(s)|} \\
& +2 \delta D \int_{t}^{s} \rho^{\prime}(\tau) e^{-\underline{c}(\rho(\tau)-\rho(t))+\varepsilon|\rho(\tau)|-3 \varepsilon|\rho(\tau)|}\|W(\tau)\| d \tau .
\end{aligned}
$$

Setting $\Gamma(t)=e^{\underline{c}(\rho(s)-\rho(t))}\|W(t)\|$ we obtain

$$
\begin{aligned}
\Gamma(t) & \leq D e^{\varepsilon|\rho(s)|}+2 \delta D \int_{t}^{s} \rho^{\prime}(\tau) e^{-2 \varepsilon|\rho(\tau)|} \Gamma(\tau) d \tau \\
& \leq D e^{\varepsilon|\rho(s)|}+2 \delta D \int_{t}^{s} \rho^{\prime}(\tau) \Gamma(\tau) d \tau .
\end{aligned}
$$

By Gronwall's lemma we have

$$
\Gamma(t) \leq D e^{\varepsilon|\rho(s)|} e^{2 \delta D(\rho(s)-\rho(t))},
$$

and thus, inequality (27) holds.

It follows from (27) and (23) that

$$
\begin{aligned}
B_{1} & \leq 2 \delta D^{2} \int_{-\infty}^{s} \rho^{\prime}(\tau) e^{(\bar{a}-\underline{c}+\varepsilon+2 \delta D)(\rho(s)-\rho(\tau))} d \tau \\
& =\frac{2 \delta D^{2}}{|\bar{a}-\underline{c}+\varepsilon+2 \delta D|}<1,
\end{aligned}
$$

provided that $\delta$ is sufficiently small. Furthermore, it follows from (26) and (24) that

$$
\begin{aligned}
B_{2} & \leq 2 \delta D^{2} \int_{s}^{\infty} \rho^{\prime}(\tau) e^{(-\underline{b}+\bar{c}+\varepsilon+2 \delta D)(\rho(\tau)-\rho(s))} d \tau \\
& =\frac{2 \delta D^{2}}{|-\underline{b}+\bar{c}+\varepsilon+2 \delta D|}<1,
\end{aligned}
$$

provided that $\delta$ is sufficiently small. This shows that $A(\phi, \Phi)$ is well-defined. Again for $\delta$ sufficiently small, it follows from (28) and (29) that

$$
\|A(\phi, \Phi)(s, \xi)\| \leq B_{1}+B_{2}<1
$$

for every $(s, \xi) \in \mathbb{R} \times E(s)$. This shows that $A(\mathcal{X} \times \mathcal{F}) \subset \mathcal{F}$.

When $(\partial f / \partial u)(t, 0)=0$ for every $t \in \mathbb{R}$, since $x_{\phi}(t, 0)=0$ for $\phi \in X$ and $t \in \mathbb{R}$, it follows from (20) and (21) that $A(\phi, \Phi)(s, 0)=0$ for every $(\phi, \Phi) \in \mathcal{X} \times \mathcal{F}_{0}$ and $s \in \mathbb{R}$. Therefore, in this case, $A\left(\mathcal{X} \times \mathcal{F}_{0}\right) \subset \mathcal{F}_{0}$.

Now we consider the transformation $S: \mathcal{X} \times \mathcal{F} \rightarrow X \times \mathcal{F}$ defined by

$$
S(\phi, \Phi)=(T \phi, A(\phi, \Phi)),
$$

with the operator $T$ as in (18). 
Lemma 6. For every sufficiently small $\delta>0$, the operator $S$ is a fiber contraction.

Proof of the lemma. Given $s \in \mathbb{R}, \xi \in E(s), \phi \in \mathcal{X}$, and $\Phi, \Psi \in \mathcal{F}$, let

$$
W_{\Phi}=W_{\phi, \Phi, \xi} \quad \text { and } \quad W_{\Psi}=W_{\phi, \Psi, \xi}
$$

Setting $\alpha=\|\Phi-\Psi\|$, we obtain

$$
\begin{aligned}
& \left\|A_{1}(\phi, \Phi)(s, \xi)-A_{1}(\phi, \Psi)(s, \xi)\right\| \\
& \leq D \int_{-\infty}^{s} e^{\bar{a}(\rho(s)-\rho(\tau))+\varepsilon|\rho(\tau)|}\left\|\frac{\partial f}{\partial x} W_{\Phi}+\frac{\partial f}{\partial y} \Phi W_{\Phi}-\frac{\partial f}{\partial x} W_{\Psi}-\frac{\partial f}{\partial y} \Psi W_{\Psi}\right\| d \tau \\
& \leq \delta D \int_{-\infty}^{s} \rho^{\prime}(\tau) e^{\bar{a}(\rho(s)-\rho(\tau))-2 \varepsilon|\rho(\tau)|}\left(\left\|W_{\Phi}-W_{\Psi}\right\|+\left\|\Phi W_{\Phi}-\Psi W_{\Psi}\right\|\right) d \tau \\
& \leq \delta D \int_{-\infty}^{s} \rho^{\prime}(\tau) e^{\bar{a}(\rho(s)-\rho(\tau))-2 \varepsilon|\rho(\tau)|} \\
& \quad \times\left(\left\|W_{\Phi}-W_{\Psi}\right\|+\|\Phi\| \cdot\left\|W_{\Phi}-W_{\Psi}\right\|+\alpha\left\|W_{\Psi}\right\|\right) d \tau \\
& \leq \delta D \int_{-\infty}^{s} \rho^{\prime}(\tau) e^{\bar{a}(\rho(s)-\rho(\tau))-2 \varepsilon|\rho(\tau)|}\left(2\left\|W_{\Phi}-W_{\Psi}\right\|+\alpha\left\|W_{\Psi}\right\|\right) d \tau
\end{aligned}
$$

and similarly,

$$
\begin{aligned}
& \left\|A_{2}(\phi, \Phi)(s, \xi)-A_{2}(\phi, \Psi)(s, \xi)\right\| \\
& \leq D \int_{s}^{\infty} e^{-\underline{b}(\rho(\tau)-\rho(s))+\varepsilon|\rho(\tau)|}\left\|\frac{\partial f}{\partial x} W_{\Phi}+\frac{\partial f}{\partial y} \Phi W_{\Phi}-\frac{\partial f}{\partial x} W_{\Psi}-\frac{\partial f}{\partial y} \Psi W_{\Psi}\right\| d \tau \\
& \leq \delta D \int_{s}^{\infty} \rho^{\prime}(\tau) e^{-\underline{b}(\rho(\tau)-\rho(s))-2 \varepsilon|\rho(\tau)|}\left(\left\|W_{\Phi}-W_{\Psi}\right\|+\left\|\Phi W_{\Phi}-\Psi W_{\Psi}\right\|\right) d \tau \\
& \leq \delta D \int_{s}^{\infty} \rho^{\prime}(\tau) e^{-\underline{b}(\rho(\tau)-\rho(s))-2 \varepsilon|\rho(\tau)|}\left(2\left\|W_{\Phi}-W_{\Psi}\right\|+\alpha\left\|W_{\Psi}\right\|\right) d \tau,
\end{aligned}
$$

where for simplicity we have omitted the arguments inside the integrals. 
In an analogous manner to that in (25) and using (26), for $t \geq s$ we have

$$
\begin{aligned}
\| & W_{\Phi}(t)-W_{\Psi}(t) \| \\
\leq & 2 \delta D \int_{s}^{t} \rho^{\prime}(\tau) e^{\bar{c}(\rho(t)-\rho(\tau))+\varepsilon|\rho(\tau)|-3 \varepsilon|\rho(\tau)|}\left\|W_{\Phi}(\tau)-W_{\Psi}(\tau)\right\| d \tau \\
& +\delta D\|\Phi-\Psi\| \int_{s}^{t} \rho^{\prime}(\tau) e^{\bar{c}(\rho(t)-\rho(\tau))+\varepsilon|\rho(\tau)|-3 \varepsilon|\rho(\tau)|}\left\|W_{\Psi}(\tau)\right\| d \tau \\
\leq & 2 \delta D e^{\bar{c}(\rho(t)-\rho(s))} \int_{s}^{t} \rho^{\prime}(\tau) e^{-\bar{c}(\rho(\tau)-\rho(s))-2 \varepsilon|\rho(\tau)|}\left\|W_{\Phi}(\tau)-W_{\Psi}(\tau)\right\| d \tau \\
& +\delta D^{2} e^{\bar{c}(\rho(t)-\rho(s))}\|\Phi-\Psi\| \int_{s}^{t} \rho^{\prime}(\tau) e^{-(\bar{c}+\varepsilon)(\rho(\tau)-\rho(s))} e^{(\bar{c}+2 \delta D)(\rho(\tau)-\rho(s))} d \tau \\
= & 2 \delta D e^{\bar{c}(\rho(t)-\rho(s))} \int_{s}^{t} \rho^{\prime}(\tau) e^{-\bar{c}(\rho(\tau)-\rho(s))-2 \varepsilon|\rho(\tau)|}\left\|W_{\Phi}(\tau)-W_{\Psi}(\tau)\right\| d \tau \\
& +\delta D^{2} e^{\bar{c}(\rho(t)-\rho(s))}\|\Phi-\Psi\| \int_{s}^{t} \rho^{\prime}(\tau) e^{-(\varepsilon-2 \delta D)(\rho(\tau)-\rho(s))} d \tau
\end{aligned}
$$

Setting

$$
\Gamma(t)=e^{-\bar{c}(\rho(t)-\rho(s))}\left\|W_{\Phi}(t)-W_{\Psi}(t)\right\|,
$$

we thus obtain

$$
\Gamma(t) \leq \frac{\delta D^{2}}{\varepsilon-2 \delta D}\|\Phi-\Psi\|+2 \delta D \int_{s}^{t} \rho^{\prime}(\tau) \Gamma(\tau) d \tau,
$$

provided that $\delta$ is sufficiently small. It follows from Gronwall's lemma that

$$
\left\|W_{\Phi}(t)-W_{\Psi}(t)\right\| \leq \frac{\delta D^{2}}{\varepsilon-2 \delta D}\|\Phi-\Psi\| e^{(\bar{c}+2 \delta D)(\rho(t)-\rho(s))} .
$$

Proceeding in a similar manner we find that for $t \leq s$,

$$
\left\|W_{\Phi}(t)-W_{\Psi}(t)\right\| \leq \frac{\delta D^{2}}{\varepsilon-2 \delta D}\|\Phi-\Psi\| e^{(-\underline{c}+2 \delta D)(\rho(s)-\rho(t))} .
$$

By (26) and (31), it follows from (30) that

$$
\begin{aligned}
& \left\|A_{1}(\phi, \Phi)(s, \xi)-A_{1}(\phi, \Psi)(s, \xi)\right\| \\
& \leq K_{1} \delta\|\Phi-\Psi\| \int_{-\infty}^{s} \rho^{\prime}(\tau) e^{(\bar{a}-\underline{c}+\varepsilon+2 \delta D)(\rho(s)-\rho(\tau))} d \tau \\
& \leq \frac{K_{1} \delta}{|\bar{a}-\underline{c}+\varepsilon+2 \delta D|}\|\Phi-\Psi\|,
\end{aligned}
$$


for some constant $K_{1}>0$, provided that $\delta$ is sufficiently small. Similarly, by $(27)$ and (32),

$$
\begin{aligned}
& \left\|A_{2}(\phi, \Phi)(s, \xi)-A_{2}(\phi, \Psi)(s, \xi)\right\| \\
& \leq K_{2} \delta\|\Phi-\Psi\| \int_{s}^{\infty} \rho^{\prime}(\tau) e^{(\bar{c}-\underline{b}+\varepsilon+2 \delta D)(\rho(\tau)-\rho(s))} d \tau \\
& \leq \frac{K_{1} \delta}{|\bar{c}-\underline{b}+\varepsilon+2 \delta D|}\|\Phi-\Psi\|,
\end{aligned}
$$

provided that $\delta$ is sufficiently small. By (33) and (34), eventually making $\delta$ sufficiently smaller, the operator $S$ becomes a fiber contraction.

Finally, we establish the continuity of the fiber contraction.

Lemma 7. For every sufficiently small $\delta>0$, the operator $S$ is continuous.

Proof of the lemma. Setting

$$
W_{\phi}=W_{\phi, \Phi, \xi} \quad \text { and } \quad W_{\psi}=W_{\psi, \Phi, \xi},
$$

we obtain

$$
\begin{aligned}
& \left\|A_{1}(\phi, \Phi)(s, \xi)-A_{1}(\psi, \Phi)(s, \xi)\right\| \\
& \leq D \int_{-\infty}^{s} e^{\bar{a}(\rho(s)-\rho(\tau))+\varepsilon|\rho(\tau)|} \\
& \quad \times \| \frac{\partial f}{\partial x}\left(y_{\phi}(\tau)\right) W_{\phi}(\tau)+\frac{\partial f}{\partial y}\left(y_{\phi}(\tau)\right) \Phi\left(z_{\phi}(\tau)\right) W_{\phi}(\tau) \\
& \quad-\frac{\partial f}{\partial x}\left(y_{\psi}(\tau)\right) W_{\psi}(\tau)-\frac{\partial f}{\partial y}\left(y_{\psi}(\tau)\right) \Phi\left(z_{\psi}(\tau)\right) W_{\psi}(\tau) \| d \tau,
\end{aligned}
$$

and

$$
\begin{aligned}
& \left\|A_{2}(\phi, \Phi)(s, \xi)-A_{2}(\psi, \Phi)(s, \xi)\right\| \\
& \leq D \int_{s}^{\infty} e^{-\underline{b}(\rho(\tau)-\rho(s))+\varepsilon|\rho(\tau)|} \\
& \quad \times \| \frac{\partial f}{\partial x}\left(y_{\phi}(\tau)\right) W_{\phi}(\tau)+\frac{\partial f}{\partial y}\left(y_{\phi}(\tau)\right) \Phi\left(z_{\phi}(\tau)\right) W_{\phi}(\tau) \\
& \quad-\frac{\partial f}{\partial x}\left(y_{\psi}(\tau)\right) W_{\psi}(\tau)-\frac{\partial f}{\partial y}\left(y_{\psi}(\tau)\right) \Phi\left(z_{\psi}(\tau)\right) W_{\psi}(\tau) \| d \tau .
\end{aligned}
$$


It follows from (7) and (27) that

$$
\begin{aligned}
& \left\|A_{1}(\phi, \Phi)(s, \xi)-A_{1}(\psi, \Phi)(s, \xi)\right\| \\
& \leq D \int_{-\infty}^{s} e^{\bar{a}(\rho(s)-\rho(\tau))+\varepsilon|\rho(\tau)|}\left\|\frac{\partial f}{\partial x}\left(y_{\phi}(\tau)\right)-\frac{\partial f}{\partial x}\left(y_{\psi}(\tau)\right)\right\| \cdot\left\|W_{\phi}(\tau)\right\| d \tau \\
& +D \int_{-\infty}^{s} e^{\bar{a}(\rho(s)-\rho(\tau))+\varepsilon|\rho(\tau)|}\left\|\frac{\partial f}{\partial x}\left(y_{\psi}(\tau)\right)\right\| \cdot\left\|W_{\phi}(\tau)-W_{\psi}(\tau)\right\| d \tau \\
& +D \int_{-\infty}^{s} e^{\bar{a}(\rho(s)-\rho(\tau))+\varepsilon|\rho(\tau)|} \\
& \quad \times\left\|\frac{\partial f}{\partial y}\left(y_{\phi}(\tau)\right)-\frac{\partial f}{\partial y}\left(y_{\psi}(\tau)\right)\right\| \cdot\left\|\Phi\left(z_{\phi}(\tau)\right) W_{\phi}(\tau)\right\| d \tau \\
& +D \int_{-\infty}^{s} e^{\bar{a}(\rho(s)-\rho(\tau))+\varepsilon|\rho(\tau)|} \\
& \quad \times\left\|\frac{\partial f}{\partial y}\left(y_{\psi}(\tau)\right)\right\| \cdot\left\|\Phi\left(z_{\phi}(\tau)\right)-\Phi\left(z_{\psi}(\tau)\right)\right\| \cdot\left\|W_{\phi}(\tau)\right\| d \tau \\
& +D \int_{-\infty}^{s} e^{\bar{a}(\rho(s)-\rho(\tau))+\varepsilon|\rho(\tau)|} \\
& \quad \times\left\|\frac{\partial f}{\partial y}\left(y_{\psi}(\tau)\right)\right\| \cdot\left\|\Phi\left(z_{\psi}(\tau)\right)\right\| \cdot\left\|W_{\phi}(\tau)-W_{\psi}(\tau)\right\| d \tau
\end{aligned}
$$

and thus,

$$
\begin{aligned}
& \left\|A_{1}(\phi, \Phi)(s, \xi)-A_{1}(\psi, \Phi)(s, \xi)\right\| \\
& \leq D^{2} e^{2 \varepsilon|\rho(s)|} \int_{-\infty}^{s} e^{(-\underline{c}+2 \delta D-\varepsilon+\bar{a})(\rho(s)-\rho(\tau))}\left\|\frac{\partial f}{\partial x}\left(y_{\phi}(\tau)\right)-\frac{\partial f}{\partial x}\left(y_{\psi}(\tau)\right)\right\| d \tau \\
& \quad+\delta D \int_{-\infty}^{s} \rho^{\prime}(\tau) e^{\bar{a}(\rho(\tau)-\rho(s))-2 \varepsilon|\rho(\tau)|}\left\|W_{\phi}(\tau)-W_{\psi}(\tau)\right\| d \tau \\
& \quad+D^{2} e^{2 \varepsilon|\rho(s)|} \int_{-\infty}^{s} e^{(-\underline{c}+2 \delta D-\varepsilon+\bar{a})(\rho(s)-\rho(\tau))}\left\|\frac{\partial f}{\partial y}\left(y_{\phi}(\tau)\right)-\frac{\partial f}{\partial y}\left(y_{\psi}(\tau)\right)\right\| d \tau \\
& \quad+\delta D^{2} \int_{-\infty}^{s} \rho^{\prime}(\tau) e^{(-\underline{c}+2 \delta D+\varepsilon+\bar{a})(\rho(s)-\rho(\tau))-\varepsilon|\rho(\tau)|}\left\|\Phi\left(z_{\phi}(\tau)\right)-\Phi\left(z_{\psi}(\tau)\right)\right\| d \tau \\
& \quad+\delta D \int_{-\infty}^{s} \rho^{\prime}(\tau) e^{\bar{a}(\rho(s)-\rho(\tau))-2 \varepsilon|\rho(\tau)|}\left\|W_{\phi}(\tau)-W_{\psi}(\tau)\right\| d \tau
\end{aligned}
$$

São Paulo J.Math.Sci. 5, 1 (2011), 1-22 


$$
\begin{aligned}
\leq & 2 D^{2} e^{2 \varepsilon|\rho(s)|} \int_{-\infty}^{s} e^{(-\underline{c}+2 \delta D-\varepsilon+\bar{a})(\rho(s)-\rho(\tau))}\left\|\frac{\partial f}{\partial u}\left(y_{\phi}(\tau)\right)-\frac{\partial f}{\partial u}\left(y_{\psi}(\tau)\right)\right\| d \tau \\
& +2 \delta D \int_{-\infty}^{s} \rho^{\prime}(\tau) e^{\bar{a}(\rho(s)-\rho(\tau))-2 \varepsilon|\rho(\tau)|}\left\|W_{\phi}(\tau)-W_{\psi}(\tau)\right\| d \tau \\
& +\delta D^{2} \int_{-\infty}^{s} \rho^{\prime}(\tau) e^{(-\underline{c}+2 \delta D+\varepsilon+\bar{a})(\rho(s)-\rho(\tau))-\varepsilon|\rho(\tau)|}\left\|\Phi\left(z_{\phi}(\tau)\right)-\Phi\left(z_{\psi}(\tau)\right)\right\| d \tau .
\end{aligned}
$$

Moreover, given $\gamma>0$ there exists $\sigma>0$ (independent of $s$ and $\xi$ ) such that, setting $\eta=\rho^{-1}(\rho(s)-\rho(\sigma))$,

$$
\begin{aligned}
& 2 D^{2} e^{2 \varepsilon|\rho(s)|} \int_{-\infty}^{\eta} e^{(-\underline{c}+2 \delta D-\varepsilon+\bar{a})(\rho(s)-\rho(\tau))}\left\|\frac{\partial f}{\partial u}\left(y_{\phi}(\tau)\right)-\frac{\partial f}{\partial u}\left(y_{\psi}(\tau)\right)\right\| d \tau \\
& \leq 4 \delta D^{2} \int_{-\infty}^{\eta} \rho^{\prime}(\tau) e^{(-\underline{c}+2 \delta D+\varepsilon+\bar{a})(\rho(s)-\rho(\tau))} d \tau \\
& =\frac{4 \delta D^{2} e^{(-\underline{c}+2 \delta D+\varepsilon+\bar{a}) \rho(\sigma)}}{|-\underline{c}+2 \delta D+\varepsilon+\bar{a}|}<\gamma \\
& 2 \delta D \int_{-\infty}^{\eta} \rho^{\prime}(\tau) e^{\bar{a}(\rho(s)-\rho(\tau))-2 \varepsilon|\rho(\tau)|}\left\|W_{\phi}(\tau)-W_{\psi}(\tau)\right\| d \tau \\
& \leq 4 \delta D^{2} \int_{-\infty}^{\eta} \rho^{\prime}(\tau) e^{(-\underline{c}+2 \delta D+\varepsilon+\bar{a})(\rho(s)-\rho(\tau))} d \tau<\gamma
\end{aligned}
$$

and

$$
\begin{aligned}
& \delta D^{2} \int_{-\infty}^{\eta} \rho^{\prime}(\tau) e^{(-\underline{c}+2 \delta D+\varepsilon+\bar{a})(\rho(s)-\rho(\tau))-\varepsilon|\rho(\tau)|}\left\|\Phi\left(z_{\phi}(\tau)\right)-\Phi\left(z_{\psi}(\tau)\right)\right\| d \tau \\
& \leq 2 \delta D^{2} \int_{-\infty}^{\eta} \rho^{\prime}(\tau) e^{(-\underline{c}+2 \delta D+\varepsilon+\bar{a})(\rho(s)-\rho(\tau))} d \tau<\gamma .
\end{aligned}
$$

Now we consider the integrals from $\eta$ to $s$. For this, setting $p=\rho(s)-\rho(\tau)$ we consider the functions

$$
\begin{aligned}
& B(p, \phi)(s, \xi)=\frac{2 D^{2} e^{2 \varepsilon|\rho(s)|} e^{(-\underline{c}+2 \delta D-\varepsilon+\bar{a}) p}}{\rho^{\prime}\left(\rho^{-1}(\rho(s)-p)\right)} \cdot \frac{\partial f}{\partial u}\left(y_{\phi}\left(\rho^{-1}(\rho(s)-p)\right)\right), \\
& C(p, \phi)(s, \xi)=2 \delta D e^{\bar{a} p-2 \varepsilon|\rho(s)-p|} W_{\phi}\left(\rho^{-1}(\rho(s)-p)\right), \\
& D(p, \phi)(s, \xi)=\delta D^{2} e^{(-\underline{c}+2 \delta D+\varepsilon+\bar{a}) p-\varepsilon|\rho(s)-p|} \Phi\left(z_{\phi}\left(\rho^{-1}(\rho(s)-p)\right)\right)
\end{aligned}
$$


for each $p \in[0, \rho(\sigma)]$ and $\phi \in X$. By (35), it is sufficient to show that the map

$$
\phi \mapsto \int_{0}^{\rho(\sigma)}[B(p, \phi)+C(p, \phi)+D(p, \phi)] d p
$$

is continuous. Since the functions $\Phi$,

$$
(t, \phi, s, \xi) \mapsto x_{\phi}(t, \xi), \quad \text { and } \quad(t, \phi, s, \xi) \mapsto W_{\phi, \Phi, \xi}(t)
$$

are continuous, the functions

$$
(p, \phi, s, \xi) \mapsto B(p, \phi)(s, \xi), C(p, \phi)(s, \xi), D(p, \phi)(s, \xi)
$$

are also continuous. Furthermore, by $(7)$ and $(27)$, for each $p \in[0, \rho(\sigma)]$ and $\phi \in \mathcal{X}$ we have

$$
\begin{aligned}
\|B(p, \phi)\| & \leq 2 \delta D^{2} e^{(-\underline{c}+2 \delta D+\varepsilon+\bar{a}) p-\varepsilon|\rho(s)-p|} \leq 2 \delta D^{2} e^{-\varepsilon|\rho(s)-p|}, \\
\|C(p, \phi)\| & \leq 2 \delta D^{2} e^{(-\underline{c}+2 \delta D+\varepsilon+\bar{a}) p-\varepsilon|\rho(s)-p|} \leq 2 \delta D^{2} e^{-\varepsilon|\rho(s)-p|}, \\
\|D(p, \phi)\| & \leq \delta D^{2} e^{(-\underline{c}+2 \delta D+\varepsilon+\bar{a}) p-\varepsilon|\rho(s)-p|} \leq \delta D^{2} e^{-\varepsilon|\rho(s)-p|},
\end{aligned}
$$

with the norm $\|\cdot\|$ in (19). In particular, $B(p, \phi), C(p, \phi)$, and $D(p, \phi)$ are in $\mathcal{F}$ provided that $\delta$ is sufficiently small. We first note that there exists $R>0$ such that

$$
\begin{gathered}
\|B(p, \phi)(s, \xi)-B(p, \psi)(s, \xi)\| \leq 4 \delta D^{2} e^{-\varepsilon|\rho(s)-p|}<\gamma, \\
\|C(p, \phi)(s, \xi)-C(p, \psi)(s, \xi)\| \leq 4 \delta D^{2} e^{-\varepsilon|\rho(s)-p|}<\gamma, \\
\|D(p, \phi)(s, \xi)-D(p, \psi)(s, \xi)\| \leq 4 \delta D^{2} e^{-\varepsilon|\rho(s)-p|}<\gamma
\end{gathered}
$$

for every $|s|>R, p \in[0, \rho(\sigma)]$, and $\xi \in E(s)$. It remains to consider the case when $|s| \leq R$. Given $s \in \mathbb{R}$ and $(\phi, \psi) \in X \times E(s)$, due to the continuity in (39) there exists $\delta>0$ such that

$$
\|B(p, \phi)(s, \xi)-B(q, \psi)(\bar{s}, \bar{\xi})\|<\gamma
$$

whenever $d(\phi, \psi)<\delta$ and $\|(p, s, \xi)-(q, \bar{s}, \bar{\xi})\|<\delta$. Since $u \mapsto f(t, u)$ vanishes for $\|u\| \geq c$, it is sufficient to establish the desired continuity for $\xi$ inside a certain ball in $E(s)$, possibly depending (continuously) on $p$ and $s$. This shows that it is sufficient to consider $\xi$ in some compact set $K$. We can cover $[0, \rho(\sigma)] \times[-R, R] \times K$ with a finite number of open balls $B_{i}$, $i=1, \ldots, r$ centered at points in this set, such that

$$
\|B(p, \phi)(s, \xi)-B(\bar{p}, \psi)(\bar{s}, \bar{\xi})\|<\gamma
$$

whenever $d(\phi, \psi)<\delta_{i}$ and $(p, s, \xi),(\bar{p}, \bar{s}, \bar{\xi}) \in B_{i}$, for $i=1, \ldots, r$ and some numbers $\delta_{i}>0$. Therefore,

$$
\|B(p, \phi)(s, \xi)-B(p, \psi)(s, \xi)\|<\gamma
$$


whenever $d(\phi, \psi)<\delta=\min \left\{\delta_{1}, \ldots, \delta_{r}\right\}$, for every $p \in[0, \rho(\sigma)],|s| \leq R$, and $\xi \in K$. This shows that

$$
\sup _{|s| \leq R} \sup _{\xi \in K}\|B(p, \phi)(s, \xi)-B(p, \psi)(s, \xi)\| \leq \gamma
$$

whenever $d(\phi, \psi)<\delta$ and $p \in[0, \rho(\sigma)]$. Similar arguments apply to $C(p, \phi)$ and $D(p, \phi)$. Together with (36), (37), and (38) this implies that $\phi \mapsto$ $A_{1}(\phi, \Phi)$ is continuous.

Similarly, it follows from (7) and (26) that

$$
\begin{aligned}
& \left\|A_{2}(\phi, \Phi)(s, \xi)-A_{2}(\psi, \Phi)(s, \xi)\right\| \\
& \leq D \int_{s}^{\infty} e^{-\underline{b}(\rho(\tau)-\rho(s))+\varepsilon|\rho(\tau)|}\left\|\frac{\partial f}{\partial x}\left(y_{\phi}(\tau)\right)-\frac{\partial f}{\partial x}\left(y_{\psi}(\tau)\right)\right\| \cdot\left\|W_{\phi}(\tau)\right\| d \tau \\
& +D \int_{s}^{\infty} e^{-\underline{b}(\rho(\tau)-\rho(s))+\varepsilon|\rho(\tau)|}\left\|\frac{\partial f}{\partial x}\left(y_{\psi}(\tau)\right)\right\| \cdot\left\|W_{\phi}(\tau)-W_{\psi}(\tau)\right\| d \tau \\
& +D \int_{s}^{\infty} e^{-\underline{b}(\rho(\tau)-\rho(s))+\varepsilon|\rho(\tau)|} \\
& \quad \times\left\|\frac{\partial f}{\partial y}\left(y_{\phi}(\tau)\right)-\frac{\partial f}{\partial y}\left(y_{\psi}(\tau)\right)\right\| \cdot\left\|\Phi\left(z_{\phi}(\tau)\right) W_{\phi}(\tau)\right\| d \tau \\
& +D \int_{s}^{\infty} e^{-\underline{b}(\rho(\tau)-\rho(s))+\varepsilon|\rho(\tau)|} \\
& \quad \times\left\|\frac{\partial f}{\partial y}\left(y_{\psi}(\tau)\right)\right\| \cdot\left\|\Phi\left(z_{\phi}(\tau)\right)-\Phi\left(z_{\psi}(\tau)\right)\right\| \cdot\left\|W_{\phi}(\tau)\right\| d \tau \\
& +D \int_{s}^{\infty} e^{-\underline{b}(\rho(\tau)-\rho(s))+\varepsilon|\rho(\tau)| \|}\left\|\frac{\partial f}{\partial y}\left(y_{\psi}(\tau)\right)\right\| \\
& \quad \times\left\|\Phi\left(z_{\psi}(\tau)\right)\right\| \cdot\left\|W_{\phi}(\tau)-W_{\psi}(\tau)\right\| d \tau .
\end{aligned}
$$

Proceeding in a similar manner to that for $A_{1}$ we find that $\phi \mapsto A_{2}(\phi, \Phi)$ is continuous, and thus $\phi \mapsto A(\phi, \Phi)$ is also continuous. This shows that the fiber contraction $S$ is continuous (we already know that the operator $T$ in (18) is a contraction, and thus it is also continuous).

To establish the $C^{1}$ regularity, we proceed with an auxiliary statement.

Lemma 8. If $\phi$ is of class $C^{1}$ in $\xi$, then $T \phi$ is of class $C^{1}$ in $\xi$, and

$$
\partial(T \phi) / \partial \xi=A(\phi, \partial \phi / \partial \xi) \text {. }
$$

Proof of the lemma. Since $\phi$ is of class $C^{1}$ in $\xi$, the function $y$ defined by $y(t, \xi)=x_{\phi}(t, \xi)$ is also of class $C^{1}$ (the right-hand side of (16) is of class $C^{1}$, and thus the solutions are $C^{1}$ in the initial conditions). Furthermore, for $\Phi=\partial \phi / \partial \xi$ the solution of equation (22) is given by $W(t)=\partial y / \partial \xi$. 
Therefore, repeating arguments in the proof of Lemma 5 we can apply Leibnitz's rule to obtain

$$
\begin{aligned}
& A(\phi, \partial \phi / \partial \xi)(s, \xi) \\
& =\left(\int_{-\infty}^{s} \frac{\partial}{\partial \xi}\left[Q_{1}(s) T(\tau, s)^{-1} f\left(\tau, x_{\phi}(\tau, \xi), \phi\left(\tau, x_{\phi}(\tau, \xi)\right)\right)\right] d \tau\right. \\
& \left.-\int_{s}^{\infty} \frac{\partial}{\partial \xi}\left[Q_{2}(s) T(\tau, s)^{-1} f\left(\tau, x_{\phi}(\tau, \xi), \phi\left(\tau, x_{\phi}(\tau, \xi)\right)\right)\right] d \tau\right) \\
& =(\partial(T \phi) / \partial \xi)(s, \xi)
\end{aligned}
$$

for every $s \in \mathbb{R}$ and $\xi \in E(s)$.

We consider the pair $\left(\phi_{1}, \Phi_{1}\right)=(0,0) \in \mathcal{X} \times \mathcal{F}$. Clearly, $\Phi_{1}=\partial \phi_{1} / \partial \xi$. We define recursively a sequence $\left(\phi_{n}, \Phi_{n}\right) \in \mathcal{X} \times \mathcal{F}$ by

$$
\left(\phi_{n+1}, \Phi_{n+1}\right)=S\left(\phi_{n}, \Phi_{n}\right)=\left(T \phi_{n}, A\left(\phi_{n}, \Phi_{n}\right)\right) .
$$

Assuming that $\phi_{n}$ is of class $C^{1}$ in $\xi$ with $\Phi_{n}=\partial \phi_{n} / \partial \xi$, it follows from Lemma 8 that $\phi_{n+1}=T \phi_{n}$ is of class $C^{1}$ in $\xi$, and by (40) we have

$$
\partial \phi_{n+1} / \partial \xi=\partial\left(T \phi_{n}\right) / \partial \xi=A\left(\phi_{n}, \Phi_{n}\right)=\Phi_{n+1} \text {. }
$$

Now let $\phi_{0}$ be the unique fixed point of $T$, and let $\Phi_{0}$ be the unique fixed point of $\Psi \mapsto A\left(\phi_{0}, \Psi\right)$. By Proposition 1 the sequences $\phi_{n}$ and $\Phi_{n}$ converge uniformly respectively to $\phi_{0}$ and $\Phi_{0}$ on bounded subsets. It follows from (42) that $\phi_{0}$ is of class $C^{1}$ in $\xi$, and that

$$
\partial \phi_{0} / \partial \xi=\Phi_{0}
$$

(we recall that if a sequence $f_{n}$ of $C^{1}$ functions converges uniformly, and the sequence $f_{n}^{\prime}$ of its derivatives also converges uniformly, then the limit of $f_{n}$ is of class $C^{1}$, and its derivative is the limit of $f_{n}^{\prime}$ ).

Now we assume that $(\partial f / \partial u)(t, 0)=0$ for every $t \in \mathbb{R}$. Since the pair $\left(\phi_{1}, \Phi_{1}\right)=(0,0)$ is in $\mathcal{X} \times \mathcal{F}_{0}$, and $S\left(\mathcal{X} \times \mathcal{F}_{0}\right) \subset \mathcal{X} \times \mathcal{F}_{0}$, the sequence $\left(\phi_{n}, \Phi_{n}\right)$ defined in (41) is also in $\mathcal{X} \times \mathcal{F}_{0}$. Therefore, $\Phi_{0}(s, 0)=0$ for every $s \in \mathbb{R}$, and it follows from (43) that in this case $\left(\partial \phi_{0} / \partial \xi\right)(s, 0)=0$ for every $s \in \mathbb{R}$.

Finally, we show that the set $V_{\phi}$ in (11) is a $C^{1}$ manifold. We already know that the function $\xi \mapsto \phi(s, \xi)$ is of class $C^{1}$ for each fixed $s \in \mathbb{R}$. We define a map

$$
F=F_{s}: \mathbb{R} \times E(s) \rightarrow \mathbb{R} \times X
$$

by

$$
F(t, \xi)=\Psi_{t}(s, \xi, \phi(s, \xi))
$$

São Paulo J.Math.Sci. 5, 1 (2011), 1-22 
Since $A$ and $f$ are of class $C^{1}$, the map

$$
\mathbb{R} \times \mathbb{R} \times X \ni(t, s, v) \mapsto \Psi_{t}(s, v)
$$

is also of class $C^{1}$, and the same happens with $F$. We can easily verify that $F$ is injective, and thus it is a parametrization of class $C^{1}$ of $V_{\phi}$. When $(\partial f / \partial u)(t, 0)=0$ for every $t \in \mathbb{R}$, the first identity in (13) yields the second one.

\section{References}

1. L. Barreira and Ya. Pesin, Nonuniform Hyperbolicity, Encyclopedia of Math. and Its Appl. 115, Cambridge Univ. Press, 2007.

2. L. Barreira and C. Valls, Smooth center manifolds for nonuniformly partially hyperbolic trajectories, J. Differential Equations 237 (2007), 307-342.

3. L. Barreira and C. Valls, Stability of Nonautonomous Differential Equations, Lect. Notes in Math. 1926, Springer, 2008.

4. L. Barreira and C. Valls, Center manifolds for nonuniform trichotomies and arbitrary growth rates, Comm. Pure Appl. Anal., to appear.

5. L. Barreira and C. Valls, Regularity of center manifolds under nonuniform hyperbolicity, preprint.

6. J. Carr, Applications of Centre Manifold Theory, Applied Mathematical Sciences 35, Springer, 1981.

7. C. Chicone, Ordinary Differential Equations with Applications, Texts in Applied Mathematics 34, Springer, 2006.

8. C. Chicone and Yu. Latushkin, Center manifolds for infinite dimensional nonautonomous differential equations, J. Differential Equations 141 (1997), 356-399.

9. $\quad$ S.-N. Chow, W. Liu and Y. Yi, Center manifolds for invariant sets, J. Differential Equations 168 (2000), 355-385.

10. S.-N. Chow, W. Liu and Y. Yi, Center manifolds for smooth invariant manifolds, Trans. Amer. Math. Soc. 352 (2000), 5179-5211.

11. S.-N. Chow and K. Lu, $C^{k}$ centre unstable manifolds Proc. Roy. Soc. Edinburgh Sect. A 108 (1988), 303-320.

12. C. Constantine and T. Savits, A multivariate Fà̀ di Bruno formula with applications, Trans. Amer. Math. Soc. 348 (1996), 503-520.

13. M. Elbialy, On sequences of $C_{b}^{k, \delta}$ maps which converge in the uniform $C^{0}$-norm, Proc. Amer. Math. Soc. 128 (2000), 3285-3290.

14. D. Henry, Geometric Theory of Semilinear Parabolic Equations, Lect. Notes in Math. 840, Springer, 1981.

15. A. Kelley, The stable, center-stable, center, center-unstable, unstable manifolds, J. Differential Equations 3 (1967), 546-570.

16. A. Mielke, A reduction principle for nonautonomous systems in infinitedimensional spaces, J. Differential Equations 65 (1986), 68-88.

17. V. Oseledets, A multiplicative ergodic theorem. Liapunov characteristic numbers for dynamical systems, Trans. Moscow Math. Soc. 19 (1968), 197-221.

18. Ya. Pesin, Families of invariant manifolds corresponding to nonzero characteristic exponents, Math. USSR-Izv. 10 (1976), 1261-1305.

19. V. Pliss, A reduction principle in the theory of stability of motion, Izv. Akad. Nauk SSSR Ser. Mat. 28 (1964), 1297-1324.

20. A. Vanderbauwhede, Centre manifolds, normal forms and elementary bifurcations, in Dynamics Reported 2, Wiley, 1989, pp. 89-169. 
21. A. Vanderbauwhede and G. Iooss, Center manifold theory in infinite dimensions, in Dynamics Reported (N.S.) 1, Springer, 1992, pp. 125-163.

22. A. Vanderbauwhede and S. van Gils, Center manifolds and contractions on a scale of Banach spaces, J. Funct. Anal. 72 (1987), 209-224.

São Paulo J.Math.Sci. 5, 1 (2011), 1-22 